ARTICLE

Received 19 Nov 2015 | Accepted 16 Apr 2016 | Published 24 May 2016

DOI: $10.1038 /$ ncomms11654

OPEN

\title{
A voltage-dependent chloride channel fine-tunes photosynthesis in plants
}

Andrei Herdean ${ }^{1}$, Enrico Teardo², Anders K. Nilsson ${ }^{1}$, Bernard E. Pfeil ${ }^{1}$, Oskar N. Johansson', Renáta Ünnep ${ }^{3,4}$,

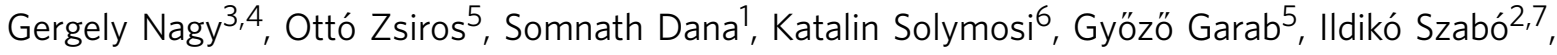
Cornelia Spetea ${ }^{1} \&$ Björn Lundin ${ }^{1}$

In natural habitats, plants frequently experience rapid changes in the intensity of sunlight. To cope with these changes and maximize growth, plants adjust photosynthetic light utilization in electron transport and photoprotective mechanisms. This involves a proton motive force (PMF) across the thylakoid membrane, postulated to be affected by unknown anion $\left(\mathrm{Cl}^{-}\right.$) channels. Here we report that a bestrophin-like protein from Arabidopsis thaliana functions as a voltage-dependent $\mathrm{Cl}^{-}$channel in electrophysiological experiments. AtVCCN1 localizes to the thylakoid membrane, and fine-tunes PMF by anion influx into the lumen during illumination, adjusting electron transport and the photoprotective mechanisms. The activity of AtVCCN1 accelerates the activation of photoprotective mechanisms on sudden shifts to high light. Our results reveal that AtVCCN1, a member of a conserved anion channel family, acts as an early component in the rapid adjustment of photosynthesis in variable light environments.

\footnotetext{
${ }^{1}$ Department of Biological and Environmental Sciences, University of Gothenburg, Gothenburg 40530, Sweden. ${ }^{2}$ Department of Biology, University of Padova, Padova 35121, Italy. ${ }^{3}$ Laboratory for Neutron Scattering and Imaging, Paul Scherrer Institute, Villigen 5232, Switzerland. ${ }^{4}$ Institute for Solid State Physics and Optics, Wigner Research Centre for Physics, Hungarian Academy of Sciences, Budapest 1121, Hungary. ${ }^{5}$ Institute of Plant Biology, Biological Research Center, Hungarian Academy of Sciences, Szeged 6701, Hungary. ${ }^{6}$ Department of Plant Anatomy, Eötvös Loránd University, Budapest 1117, Hungary. ${ }^{7}$ CNR Neuroscience Institute, Padova 35121, Italy. Correspondence and requests for materials should be addressed to C.S. (email: cornelia.spetea.wiklund@bioenv.gu.se) or to B.L. (email: bjorn.lundin@gmail.com).
} 
C hloroplasts have essential roles in harvesting and converting energy from the sun into carbohydrates, which are then used in cell metabolism. The protein machineries in the two aqueous compartments of this organelle (stroma and thylakoid lumen) are fine-tuned to the demands of the cell by changes in ion balance ${ }^{1}$. Photosynthetic electron transport in thylakoid membranes and the architecture of these membranes are highly sensitive to the concentration of ions $\left(\mathrm{H}^{+}, \mathrm{K}^{+}, \mathrm{Mg}^{2+}\right.$ and $\mathrm{Cl}^{-}$) in the stroma and thylakoid lumen ${ }^{1,2}$. In natural habitats, plants experience variable light conditions, for example, shifts in light intensity and quality within seconds to minutes due to clouds, canopy architecture and leaf movement due to wind. To adjust to variable light, rapid changes in ion balance of the chloroplast occur through the regulation of ion transport ${ }^{3}$.

Ion channel activities across chloroplast envelopes and thylakoid membranes have been demonstrated, and are postulated to play critical roles in chloroplast physiology ${ }^{4-7}$. Light-induced charge separation and coupled $\mathrm{H}^{+}$uptake into the thylakoid lumen generate a proton motive force $(\mathrm{PMF})$, composed of the transmembrane electric-potential gradient $(\Delta \Psi)$ and $\mathrm{H}^{+}$ concentration gradient $(\Delta \mathrm{pH})$. Both PMF components activate and drive ATP synthesis by chloroplast $\mathrm{F}_{0} \mathrm{~F}_{1}$ ATP synthase. A high $\mathrm{H}^{+}$concentration in the thylakoid lumen downregulates electron transport at the level of the cytochrome $b_{6} f$ complex and activates photoprotection through the dissipation of excess light as heat (non-photochemical quenching, NPQ) ${ }^{8}$. PMF partitioning into $\Delta \mathrm{pH}$ and $\Delta \Psi$ can vary with changes in the light environment, and is proposed to be a fine-tuning mechanism for photosynthesis? More specifically, the plant stores PMF predominantly as $\Delta \mathrm{pH}$ to downregulate electron transport and rapidly activate NPQ in conditions of sudden increases in light intensity, which would otherwise result in damage to the photosynthetic machinery and reduction in growth ${ }^{10}$. Conversely, the plant reduces the fraction of PMF stored as $\Delta \mathrm{pH}$ after transitions to low light intensities to downregulate NPQ and maximize photosynthesis and growth. The mechanism by which PMF is partitioned and fine-tuned to achieve rapid photosynthetic acclimation is poorly understood. A critical factor is thought to be the ionic composition of the stroma and thylakoid lumen. The reasoning is that fast movements of counterions $\left(\mathrm{Cl}^{-}\right.$influx, $\mathrm{Mg}^{2+}$ and $\mathrm{K}^{+}$efflux) electrically balance $\mathrm{H}^{+}$uptake into the thylakoid lumen ${ }^{4}$. Hence, these ion fluxes would rapidly adjust PMF partitioning by efficient regulation of $\Delta \Psi$.

Our recent study in Arabidopsis thaliana ${ }^{11}$ revealed that a thylakoid $\mathrm{K}^{+}$channel (AtTPK3) is involved in the modulation of PMF partitioning into $\Delta \Psi$ and $\Delta \mathrm{pH}$ through $\mathrm{K}^{+}$efflux from the lumen, and in this way controls utilization of light in photosynthesis. More recently, two laboratories independently localized a $\mathrm{K}^{+} / \mathrm{H}^{+}$exchanger (AtKEA3) to thylakoids and showed that it modulated PMF partitioning through $\mathrm{H}^{+}$efflux from the lumen, which is critical for photosynthetic acclimation after transitions from high to low light ${ }^{3,12}$. In comparison with $\mathrm{K}^{+}$ transport, knowledge about the $\mathrm{Cl}^{-}$fluxes that counterbalance $\mathrm{H}^{+}$pumping in thylakoids is still limited, even though this is necessary to fully understand the mechanism of PMF partitioning by ions. Activities of voltage-dependent $\mathrm{Cl}^{-}$channels have been reported in plant and algal thylakoid membranes ${ }^{6,7,13}$. However, they have not been conclusively attributed to any specific proteins. A member of the $\mathrm{Cl}^{-}$channel family $\mathrm{ClC}$ in Arabidopsis, named AtClCe, was localized to the thylakoid membrane ${ }^{14-16}$. This protein was initially proposed to be involved in photosynthesis ${ }^{15}$, and later in $\mathrm{NO}_{3}^{-}$assimilation pathways ${ }^{17}$. Whether $\mathrm{ClCe}$ works as a $\mathrm{Cl}^{-}$channel remains to be demonstrated. In this work, we describe a voltage-dependent $\mathrm{Cl}^{-}$channel located in Arabidopsis thylakoids (AtVCCN1), where it functions to fine-tune PMF and allows the plant to adjust photosynthesis to variable light.

\section{Results}

AtVCCNs are thylakoid members of a conserved channel family. A T-DNA insertion mutant of AtVCCN1 gene locus (At3g61320) was annotated to have altered NPQ by the Chloroplast Phenomics 2010 project (www.plastid.msu.edu) ${ }^{18}$, which identified previously uncharacterized regulatory photosynthetic proteins ${ }^{19,20}$. This gene encodes a chloroplast-predicted membrane protein, which was annotated as 'bestrophin-like' in public databases. Bestrophins function as $\mathrm{Ca}^{2+}$-activated monovalent anion channels in mammalian membranes ${ }^{21}$.

Data extracted at ARAMEMNON database (http://aramemnon.botanik.uni-koeln.de/ $/)^{22}$ indicated that the amino-acid sequence of AtVCCN1 comprises a predicted chloroplasttargeting peptide (residues 1-40), 4-5 putative transmembrane domains and termini of 60-70 residues (Supplementary Fig. 1). Using phylogenetic analyses, we found that the protein sequence was highly conserved in land plants, and that it had homologues in cyanobacteria, red and green algae, diatoms and haptophytes (Supplementary Fig. 2, Supplementary Data 1). The fact that we found homologues in non-photosynthetic bacteria and eukaryotes (fungi and arthropods) suggests a widespread importance of the biochemical function of VCCNs. No mammalian bestrophins were found in our searches, and alignment to human BEST1 revealed only 9\% identity. Three residues important for anion selectivity in bestrophins ${ }^{21}$ were conserved, but four out of five acidic residues involved in $\mathrm{Ca}^{2+}$ binding were missing in AtVCCN1 (Supplementary Fig. 1). This casts doubt on the previous annotation as a 'bestrophin-like protein', and instead suggests a family of uncharacterized anion channels. Similar to other thylakoid transport proteins such as $\mathrm{ClCe}^{23}$, an origin of this family from the host or symbiont in the endosymbiotic event in the evolution of chloroplasts could not be discerned in our analyses.

A close paralogue of AtVCCN1 is the protein encoded by the At2g45870 gene, which we named AtVCCN2. Their amino-acid sequences are highly similar (76\% identity; $86 \%$ similarity) and diverged during the early evolution of the Brassicaceae (c. 24-40 million years ago), but they are part of distinct clades (Supplementary Fig. 3a). AtVCCN2 also has a predicted chloroplasttargeting peptide, but we found that its transcripts were more abundant in flowers than in leaves, in contrast to AtVCCN1 transcripts that were highly abundant both in leaves and flowers (Supplementary Fig. 3b,c), which was in agreement with Genevestigator database (https://genevestigator.com/) ${ }^{24}$.

For intracellular localization in Arabidopsis, we stably introduced green fluorescent protein (GFP) fusions of AtVCCN1 and AtVCCN2 into corresponding knockout mutants ( $v c c n 1-1$ and $v c c n 2-1$, respectively) and overexpressed them under the control of CaMV 35S constitutive promoter. In leaf protoplasts isolated from transformed plants, the GFP fluorescence signal of both fusions colocalized with chlorophyll fluorescence of chloroplasts (Fig. 1a), thereby confirming the predicted chloroplast location. Immunoblotting of chloroplast subfractions isolated from leaves of transformed plants revealed a location for both AtVCCNs in the thylakoid membrane, more precisely in the stroma-exposed regions (Fig. 1b,c, Supplementary Fig. 4a,b). Due to the lack of specific antibodies against the two proteins, the expression level of AtVCCN1 and AtVCCN2 proteins in non-transformed wild-type (wt) plants could not be assessed. However, on the basis of the tissue expression pattern, it is likely that AtVCCN1 is the predominant form in thylakoids from wt leaves.

Electrophysiology of AtVCCN1 in planar lipid bilayers. Previous electrophysiological studies on thylakoid membranes have revealed the activity of two distinct anion channels, one with 
a

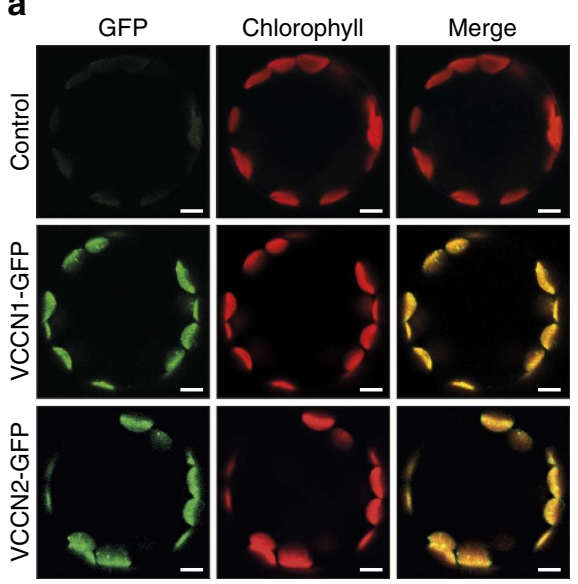

b

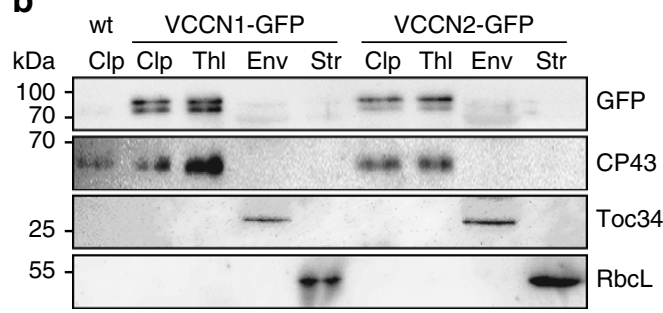

C

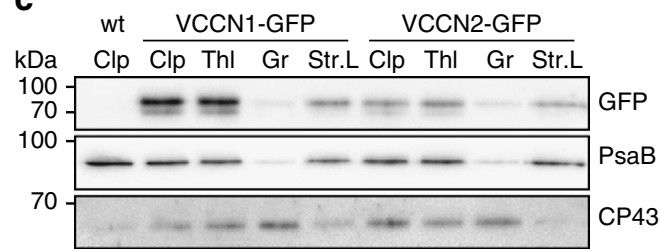

Figure 1 | Chloroplast localization of VCCN1 and VCCN2 in Arabidopsis. (a) GFP and chlorophyll fluorescence imaged in leaf protoplasts from Arabidopsis wild-type plants (control) and vecn mutants transformed with AtVCCN-GFP fusions. Scale bars, $20 \mu \mathrm{m}$. (b,c) Localization of AtVCCN1 and AtVCCN2 in chloroplast and thylakoid subfractions by immunoblotting with an anti-GFP antibody. Chloroplasts (Clp), envelope (Env), stroma (Str), thylakoids (Thl), grana (Gr) and stroma lamellae (Str.L) were purified from leaves of plants transformed with AtVCCN1-GFP or AtVCCN2-GFP. Purity of the fractions was validated using antibodies against marker proteins for the respective compartments: chloroplast outer envelope membrane translocon complex Toc34 protein, ribulose bisphosphate carboxylase large subunit $\mathrm{RbcL}$, photosystem I subunit PsaB and photosystem II subunit CP43.

a conductance of $110 \mathrm{pS}$ in $110 \mathrm{mM} \mathrm{KCl}^{7,13}$ and another one with a conductance of $220 \mathrm{pS}$ in $100 \mathrm{mM} \mathrm{KCl}^{6}$. We decided to study the biophysical function of AtVCCN1 in a recombinant form expressed in an in vitro transcription/translation system previously used for the study of various ion channels ${ }^{25-27}$ (Supplementary Fig. 5). When inserted into a planar lipid bilayer, the AtVCCN1 protein reproducibly displayed a channel activity in $\mathrm{Cl}^{-}$and $\mathrm{NO}_{3}^{-}$media (Fig. 2a, Supplementary Figs $6,7 \mathrm{~d}, 8 \mathrm{a}-\mathrm{c}, 9 \mathrm{a})$. The channel was selective for $\mathrm{Cl}^{-}$over $\mathrm{K}^{+}$displaying a permeability ratio of $P_{\mathrm{Cl}}^{-}: P_{\mathrm{K}}^{+}=1: 0.17 \pm 0.03$ ( \pm indicates s.d., $n=3$ ), as determined from the reversal potential under asymmetric ionic conditions $(300 \mathrm{mM} / 100 \mathrm{mM}$ $\mathrm{KCl}$; Supplementary Fig. 9), and calculated according to the Goldman-Hodgkin-Katz equation ${ }^{28}$. The single-channel conductance of AtVCCN1 in $100 \mathrm{mM} \mathrm{KCl}(96.1 \pm 5.0 \mathrm{pS}$ and $60.0 \pm 8.2 \mathrm{pS}$ at positive and negative voltages, respectively, where \pm indicates s.d., $n=5)$ was higher than that in $100 \mathrm{mM}$ $\mathrm{KNO}_{3}(28.7 \pm 12.2 \mathrm{pS}$ at both voltage ranges, where \pm indicates s.d., $n=3$; Fig. 2a, Supplementary Figs $7 c, 8 d$ ), indicating a preference for $\mathrm{Cl}^{-}$over $\mathrm{NO}_{3}^{-}$. The channel displayed voltage dependence, being more active at positive than at negative voltages (Fig. 2a, Supplementary Figs 6,9,10). A fast-gating, flickering pattern was observed at positive voltages, whereas burst-like openings were dominant at negative voltages (Fig. 2a, Supplementary Figs 8a,10b). Furthermore, cooperative gating, that is, simultaneous opening of more than one channel and conductance sub-states (Fig. 2a, Supplementary Figs 8b,9) were observed frequently, as were changes in kinetic behaviour, that is, from slow to fast gating (Supplementary Fig. 8c). AtVCCN1 activity was blocked by general anion channel inhibitors dithiocyanatostilbene-2,2' -disulphonic acid (DIDS) and niflumic acid (Fig. 2b, Supplementary Figs 6c,7a,b,8e). Activity was affected by neither the $\mathrm{K}^{+}$channel inhibitor tetraethylammonium (Supplementary Fig. 8f) nor by $\mathrm{Ca}^{2+}$ (Supplementary Fig. 7d), in accordance with the absence of $\mathrm{Ca}^{2+}$-binding motifs in the AtVCCN1 sequence (Supplementary Fig. 1). Taken together, these data demonstrate that AtVCCN1 forms a voltage-dependent, DIDS-sensitive and anion-selective channel that is permeable to $\mathrm{Cl}^{-}$and $\mathrm{NO}_{3}^{-}$. These characteristics are similar to those of the channel recorded directly in the thylakoid membrane of Peperomia metallica by
Schönknecht et al. ${ }^{7}$ and in Nitellopsis obtusa by Pottosin and Schönknecht ${ }^{13}$. There are also differences from the previously reported activity: AtVCCN1 is less permeable to $\mathrm{NO}_{3}^{-}$than to $\mathrm{Cl}^{-}$, is also active at high voltages, and displays a different gating behaviour. The use of different species with respect to the above-mentioned works, different lipid composition and/or lack of possible regulatory subunits, post-translational modification in our system might a priori cause such differences. However, it cannot be excluded that AtVCCN1 and the proteins giving rise to the activities thus far characterized are distinct. The conductance and overall characteristics of AtVCCN1 were significantly different from those of another anion channel observed in thylakoids by Wagner and colleagues ${ }^{6}$.

PMF and photosynthetic acclimation from dark to light. In the thylakoid membrane, the above-described activity for AtVCCN1 by electrophysiology would allow immediate $\mathrm{Cl}^{-}$influx at the onset of illumination in response to an increase in thylakoid membrane voltage, that is, the $\Delta \Psi$ component of PMF (positive on lumenal side) created by $\mathrm{H}^{+}$uptake. $\mathrm{Cl}^{-}$influx would in turn contribute to the rapid reduction in $\Delta \Psi$ resulting in higher $\Delta \mathrm{pH}$. Conversely, defects in $\mathrm{Cl}^{-}$influx would allow the total PMF to be higher and stored more as $\Delta \Psi$ at the expense of $\Delta \mathrm{pH}$. The altered PMF size and partitioning are expected to perturb $\mathrm{H}^{+}$efflux through ATP synthase, electron transport and activation of NPQ, with consequences for the acclimation of the plant after transition from dark to light and in fluctuating light conditions.

To investigate the role of AtVCCN1 activity in these processes in leaves, we characterized two Arabidopsis T-DNA insertion knockout lines (vccn1-1, vccn1-2) and the overexpression line used in the localization of AtVCCN1-GFP (oeVCCN1; Supplementary Fig. 11). All genotypes grew like wt in standard conditions and displayed similar levels of photosynthetic proteins and pigments (Supplementary Fig. 12, Supplementary Table 1). To determine the total PMF size, we recorded decay of the total electrochromic shift $\left(\mathrm{ECS}_{\mathrm{t}}\right)$ by applying brief $(600 \mathrm{~ms})$ dark intervals at specific time points during illumination of intact leaves. PMF size and its partitioning into $\Delta \Psi$ and $\Delta \mathrm{pH}$ were subsequently determined from the dark interval decay kinetics of the ECS signal (Supplementary Fig. 13a,b). On transition from 
a

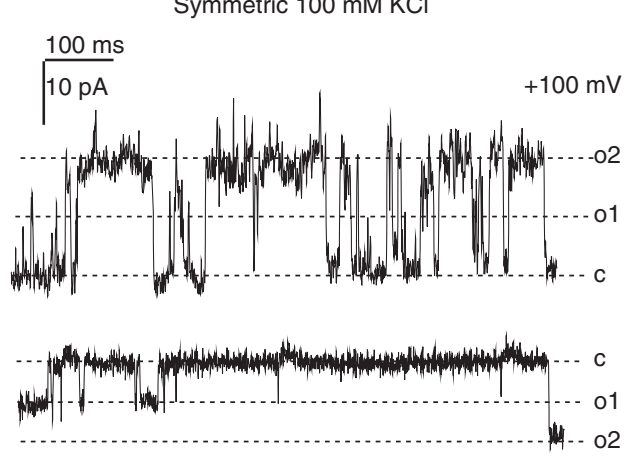

Symmetric $100 \mathrm{mM} \mathrm{KNO}_{3}$
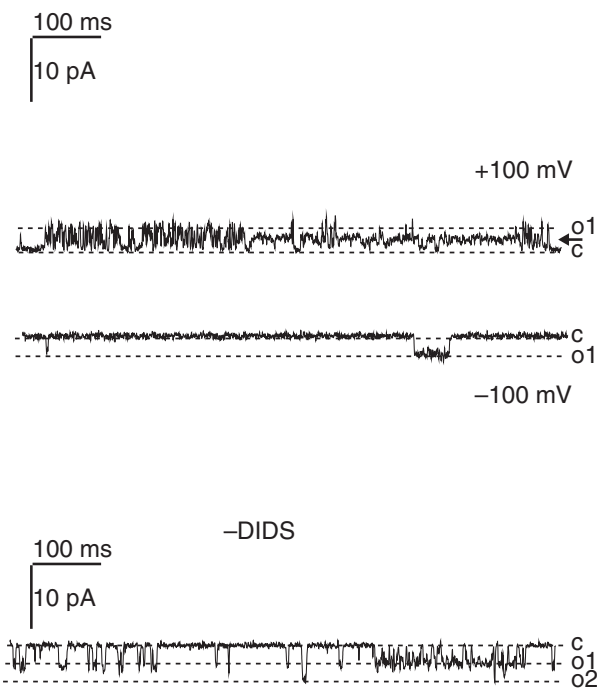

+DIDS

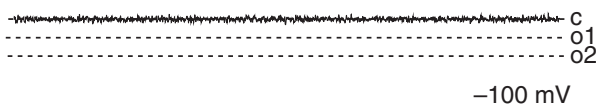

b

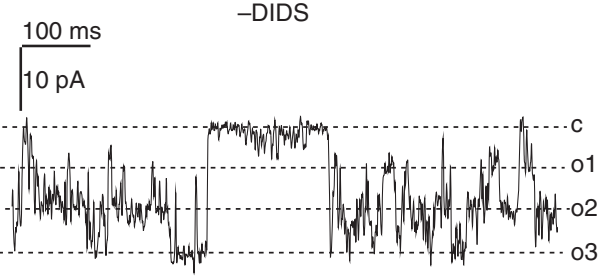

+DIDS

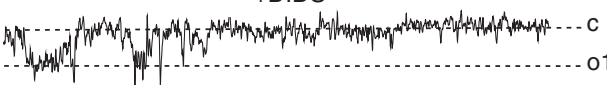

01

(1)

$-100 \mathrm{mV}$

Figure 2 | Recombinant AtVCCN1 displays a voltage-sensitive anion channel activity in planar lipid bilayers. (a) Current traces recorded in symmetric $\mathrm{KCl}$ (representative of 15 experiments) or $\mathrm{KNO}_{3}$ (representative of 26 experiments) solutions (cis side: $100 \mathrm{mM}$, trans side: $100 \mathrm{mM}$ ) at indicated potentials (cis side) are shown (c, closed state; 01, 02, 03, open states). The arrow in the upper right panel indicates a subconductance state. (b) Current traces recorded before and after addition of $20 \mu \mathrm{M}$ DIDS (left: $100 \mathrm{mM} \mathrm{KCl}, n=4$; right: $100 \mathrm{mM} \mathrm{KNO}_{3}, n=6$; see also Supplementary Fig. $6 \mathrm{c}$; for amplitude histograms, see Supplementary Figs 7a,8e). The general anion channel blocker DIDS caused inhibition of the activity in accordance with AtVCCN1 being an anion channel.

dark to light, PMF reached maximal values within the first 2 min, decreased by $20 \%$ until $5 \mathrm{~min}$ and remained stable for the remaining illumination time in wt plants (Fig. 3a). In vccn1-1, PMF size was moderately but significantly elevated, whereas in oeVCCN1 it was strongly reduced as compared with wt. A significantly enhanced capacity to maintain high $\Delta \Psi$ at the expense of $\Delta \mathrm{pH}$ was observed in $v c c n 1-1$ at both studied time points (Fig. 3b). The residual PMF observed in oeVCCN1 consisted entirely of $\Delta \mathrm{pH}$, indicating the behaviour of a ' $\Delta \Psi$ uncoupler' for the overexpressed protein. These data are consistent with our hypothesis described above, that is, that AtVCCN1 is immediately activated on illumination and dissipates part of $\Delta \Psi$ to maintain a significant $\Delta \mathrm{pH}$ across the thylakoid membrane. A complementary method using $\mathrm{pH}$-sensitive acridine orange (AO) fluorescence in isolated chloroplasts indicated significantly more acidic lumenal $\mathrm{pH}$ in the light in oeVCCN1 than in wt (Supplementary Fig. 14), supporting the higher PMF partitioning to $\Delta \mathrm{pH}$ in this line. In contrast to the more acidic lumenal $\mathrm{pH}$, the total PMF size was lower than in wt (Fig. 3a). We do not exclude the possibility that PMF size was underestimated in oeVCCN1, for example, because of elevated accumulation of negative charges $\left(\mathrm{Cl}^{-}\right)$in the thylakoid lumen. This may have prevented the decay of the ECS signal on turning off the light during ECS measurement, which is required for estimation of PMF. This possibility does not alter the interpretation of our results for the other genotypes where decay of the ECS signal clearly takes place (Supplementary Fig. 13a,b).
Next, we determined the rate of $\mathrm{H}^{+}$efflux through the thylakoid membrane (mainly via the ATP synthase) $\left(\mathrm{g}_{\mathrm{H}}^{+}\right)$ from ECS decay during $600 \mathrm{~ms}$ dark intervals (Supplementary Fig. $13 \mathrm{c}, \mathrm{d})$. The observed rate was higher in $v c c n 1-1$ than in wt throughout the illumination period (Fig. 3c), and resembled the pattern observed for PMF size rather than PMF partitioning (Fig. 3a,b). This result is in accordance with the knowledge that $\mathrm{H}^{+}$efflux through the ATP synthase can be driven by both PMF components and is dependent on the total PMF size ${ }^{8}$. A reduced rate of $\mathrm{H}^{+}$efflux was observed in oeVCCN1 in the first minute of illumination as compared with wt (Fig. 3c). Later on during illumination, the rate increased over that of wt despite the reduced total PMF size. In control experiments, we incubated the leaves with $\mathrm{N}, \mathrm{N}$-dicyclohexylcarbodiimide (DCCD), which binds covalently to an $\mathrm{F}_{0}$-subunit, thus inhibiting $\mathrm{H}^{+}$efflux through the ATP synthase ${ }^{29}$. DCCD reduced the rate of $\mathrm{H}^{+}$ efflux in both wt and mutants to similar levels (Supplementary Fig. 15), confirming that $\mathrm{H}^{+}$efflux through the ATP synthase caused the observed differences between the three genotypes.

Chlorophyll fluorescence measurements were performed to determine the effects of AtVCCN1 on the regulation of photosynthesis. NPQ transiently decreased in $v c c n 1$ and increased in oeVCCN1, resulting in an adjustment of the linear electron transport measured as PSII quantum yield ( $\Phi_{\mathrm{II}}$; Fig. $3 \mathrm{~d}, \mathrm{e}$, Supplementary Fig. 16). On the basis of these data and the electrophysiological results described above, we suggest that AtVCCN1 is involved in light-triggered voltage-dependent $\mathrm{Cl}^{-}$ fluxes in thylakoids, which contribute to the counterbalancing of 
$\mathrm{H}^{+}$pumping. In this way, AtVCCN1 fine-tunes the PMF and photosynthesis on transition from dark to light.

Although less abundant than AtVCCN1, AtVCCN2 is also expressed in leaves (Supplementary Fig. 3b,c), and may share a location in the chloroplast thylakoid membrane (Fig. 1b,c). To address possible functional redundancy between the two proteins, we characterized vccn2, vccn $1 v c c n 2$ and oeVCCN2 (Supplementary Figs 11,12 and Supplementary Table 1) and compared them with wt, $v c c n 1$ and oeVCCN1. We show that
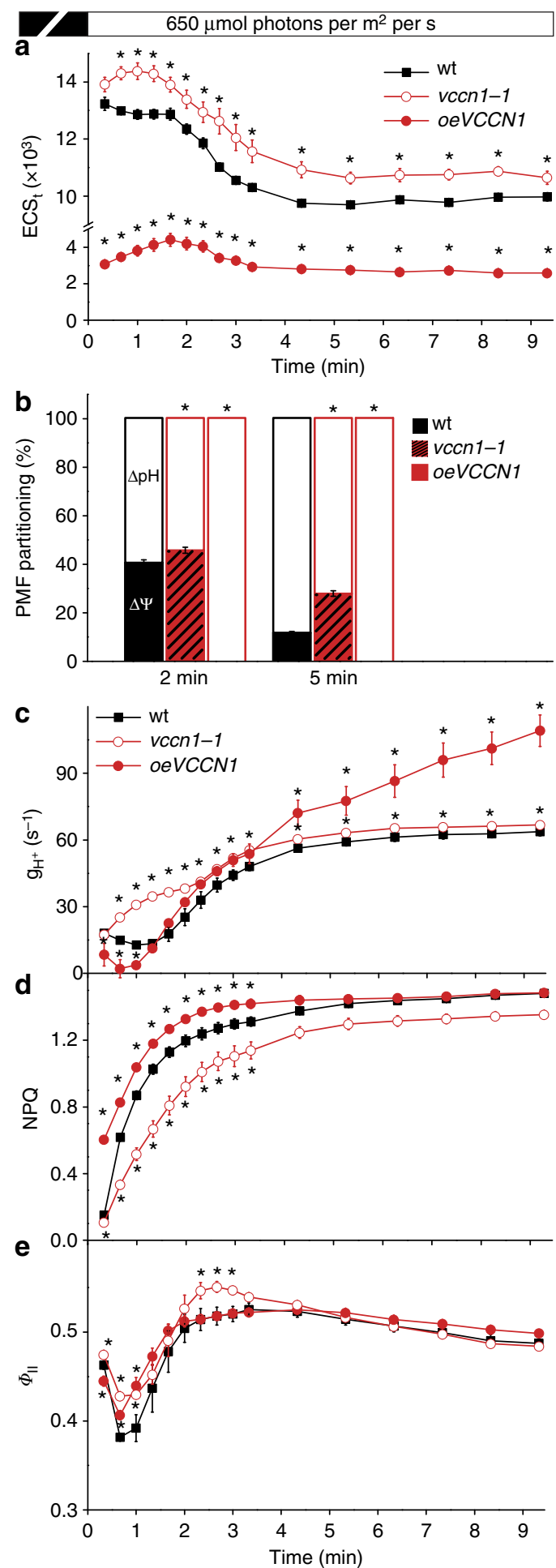

$v c c n 2$ displayed a wt-like pattern for the measured parameters, whereas $v c c n 1 v c c n 2$ closely resembled $v c c n 1$ (Supplementary Figs 17,18). Interestingly, the oeVCCN2 line displayed a wt phenotype in PMF size, partitioning and rate of $\mathrm{H}^{+}$efflux in the first 2-3 min of illumination, and resembled oeVCCN1 afterwards (Supplementary Fig. 19). Pre-treatment with DCCD reduced the rate of $\mathrm{H}^{+}$efflux through ATP synthase to similar levels in all six genotypes (Supplementary Fig. 15). These data suggest that AtVCCN2 is not redundant to AtVCCN1 in terms of the regulation of PMF and photosynthesis in leaf thylakoids. However, AtVCCN2 could form a channel based on the sequence similarity (Supplementary Fig. 1). Indeed, overexpressed AtVCCN2 triggered a similar response as overexpressed AtVCCN1 but with different kinetics.

Photosynthetic acclimation from low to high light. To further investigate the role of AtVCCN1 in photosynthetic regulation, we undertook experiments under fluctuating light. As for the dark-to-light transition, $v c c n$ 1-1 displayed slower NPQ induction, but without significant change in linear electron transport compared with wt (Fig. 4a,b). In oeVCCN1, NPQ was induced faster and decayed slower, resulting in less electron transport than seen in the wt. This pattern of changes in vccn1-1 and oeVCCN1 can be explained by the observation that AtVCCN1 activity favours the build-up of $\Delta \mathrm{pH}$ (Fig. 3b), and suggests its involvement in fine-tuning photosynthesis on transitions from low to high light. On the basis of the kinetic pattern of the parameters presented in Figs $3 \mathrm{a}-\mathrm{e}$ and $4 \mathrm{a}$, b we propose a model for the sequence of events where AtVCCN1 plays role in the regulation of photosynthesis (Fig. 4c).

AtVCCN1 influences thylakoid ultrastructure. In addition to charge compensation of light-driven $\mathrm{H}^{+}$uptake in thylakoids, ion fluxes have been hypothesized to modulate the overall architecture of the thylakoid membrane because the formation of thylakoid stacks (grana) is known to depend on the ionic strength $^{1,2}$. An impaired anionic permeability in $v c c n 1$ would result in changes in intra-thylakoid ionic status and would alter thylakoid ultrastructure. Transmission electron microscopic (TEM) analyses revealed significantly longer grana in vccn1-1 than in wt plants in the dark-adapted state (Fig. 5, Supplementary Table 2). In light-adapted plants, vccn1-1 grana were also longer than in wt and in addition, often exhibited a curved 'banana-like' shape rather than the horizontal shape that was commonly observed in the other samples. The overall increased granum size and modified shape in vccn1-1 suggests significant rearrangements of the thylakoid membrane network as a result of altered ionic strength.

Figure 3 | Kinetics of proton motive force and photosynthetic acclimation on transition from dark to light. (a) Kinetics of $E C S_{t}$ (total electrochromic shift, a measure of total proton motive force size, PMF) in wild-type plants (wt), vccn1-1 knockout mutant and mutant overexpressing AtVCCN1 (oeVCCN1) were recorded at $650 \mu \mathrm{mol}$ photons per $\mathrm{m}^{2}$ per $\mathrm{s}$ after $30 \mathrm{~min}$ dark adaptation. (b) Fraction of PMF contributed by transmembrane electric-potential gradient $(\Delta \Psi)$ and $\mathrm{H}^{+}$concentration gradient $(\Delta \mathrm{pH})$ at two time points in the light. (c) $\mathrm{H}^{+}$conductivity through ATP synthase $\left(\mathrm{g}_{+}^{+}\right)$was determined from ECS decay kinetics. (d) Kinetics for induction of non-photochemical quenching (NPQ) as a measure of photoprotective mechanisms were recorded during $10 \mathrm{~min}$ of illumination at $650 \mu \mathrm{mol}$ photons per $\mathrm{m}^{2}$ per $\mathrm{s}$. (e) Photosystem II quantum yield $\left(\Phi_{\|}\right)$as a measure of linear electron transport was calculated from the same experiment as NPQ. Data are the means \pm s.e.m. $(n=5)$. Where not visible, error bars are smaller than the symbols. Asterisks denote a statistically significant difference between wt and mutants (Student's $t$-test, $P<0.05$ ). 


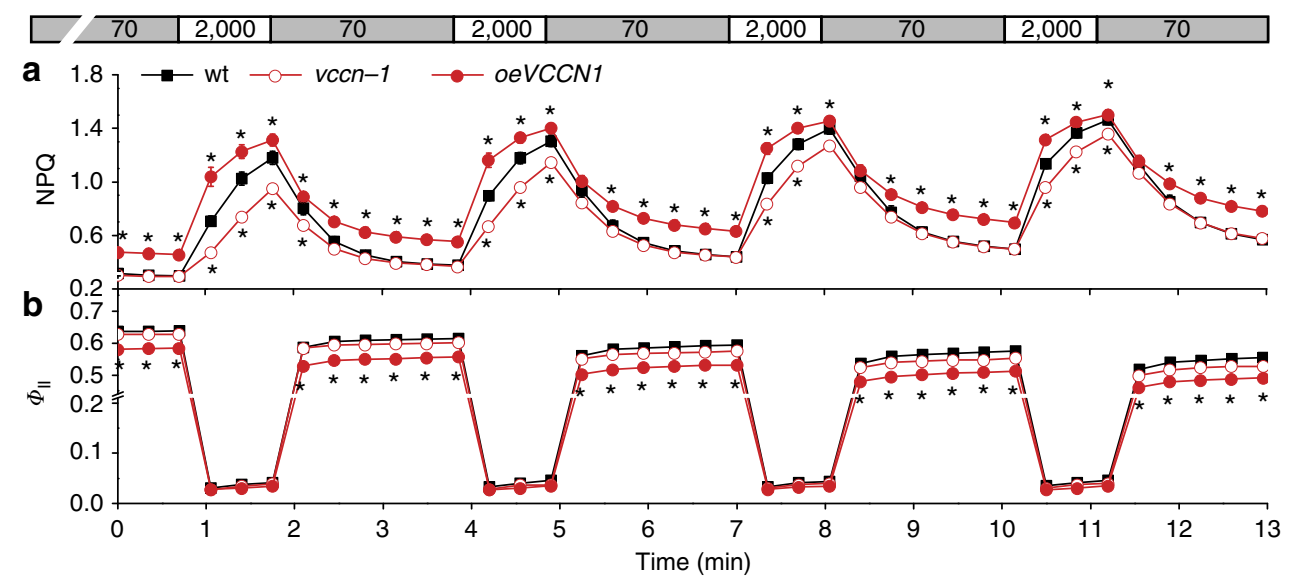

C

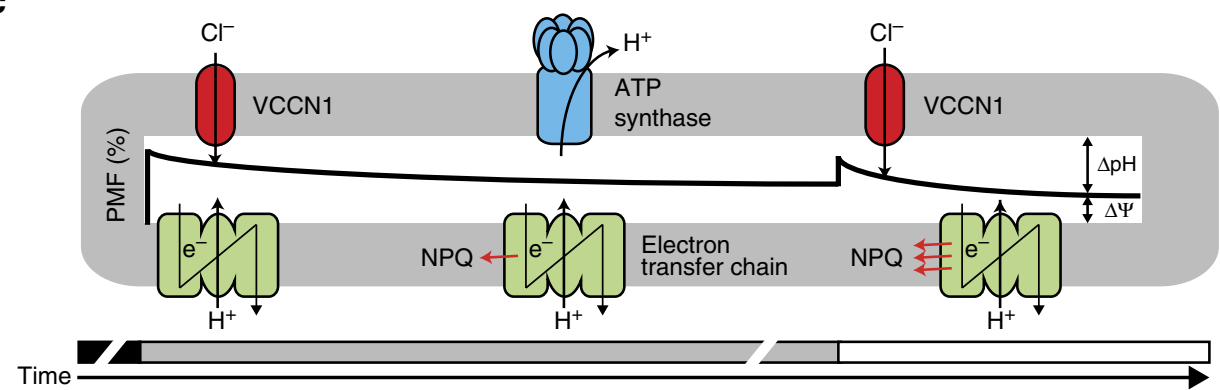

Figure 4 | Dynamics of photosynthesis and photoprotection during transitions from low to high light. (a) Induction of non-photochemical quenching (NPQ) is slowed down in vccn1-1 but approaches wild-type (wt) levels with each transition from low light ( $70 \mu$ mol photons per $\mathrm{m}^{2}$ per $\mathrm{s}$ ) to high light $\left(2,000 \mu \mathrm{mol}\right.$ photons per $\mathrm{m}^{2}$ per s). NPQ is induced faster in oeVCCN1 and decays slower than in wt. (b) Photosystem II quantum yield ( $\left.\Phi_{\|}\right)$as a measure of electron transport is not changed in vccn1-1, but is reduced in oeVCCN1 when compared with the wt. Data are the means \pm s.e.m. $(n=5)$. Where not visible, error bars are smaller than the symbols. Asterisks denote a statistically significant difference between wt and mutants (Student's $t$-test, $P<0.05$ ). (c) Simplified model for sequence of events in the regulation of photosynthesis by AtVCCN1. At the onset of illumination (grey bar), electron transportcoupled $\mathrm{H}^{+}$pumping into the thylakoid lumen results in the formation of proton motive force (PMF), consisting of a major transmembrane electricpotential gradient $(\Delta \Psi)$ and a minor $\mathrm{pH}$ gradient $(\Delta \mathrm{pH})$. AtVCCN1 is activated by $\Delta \Psi$ and partially dissipates it by anion influx. This phenomenon allows a rapid increase in $\Delta \mathrm{pH} / \Delta \Psi$ ratio, which is maintained during the remaining illumination. AtVCCN1 also fine-tunes the size of total PMF (not illustrated in this model), which in turn regulates $\mathrm{H}^{+}$efflux from the thylakoid lumen through ATP synthase. Electron transport and activation of NPQ are modulated by the $\Delta \mathrm{pH} / \Delta \Psi$ ratio. After sudden shift from low light to high light (white bar), the $\Delta \mathrm{pH} / \Delta \Psi$ ratio is changed by an increase in $\Delta \Psi$, but AtVCCN1 reestablishes a steady-state, resulting in rapid adjustment of photosynthesis.

Studies using non-invasive small-angle neutron scattering (SANS) on detached, deuterium oxide $\left(\mathrm{D}_{2} \mathrm{O}\right)$-infiltrated leaves from dark-adapted $v c c n 1-1$ indicated a shift of the Bragg peak related to periodicity of the thylakoid stacks (grana) to higher $q$ values as compared with wt (Supplementary Fig. 20a). This shift revealed a smaller lamellar repeat distance (RD) of grana in vccn1-1 (Supplementary Table 2), which we attribute to a tighter packing than in wt. Isolated thylakoid membranes, while exhibiting larger RDs than leaves ${ }^{30}$, retained the small difference between the two genotypes (Supplementary Fig. 20b, Supplementary Table 2). On addition of $\mathrm{KCl}$ or $\mathrm{KNO}_{3}$, slight shrinkage of grana occurred in both samples, as indicated by a minor shift in the Bragg peak to higher $q$ values, accompanied by a decrease in the Bragg peak-with this, the RD in the wt decreased to that of untreated vccn1-1 (Supplementary Fig. 20c,d).

Circular dichroism (CD) spectra on detached leaves revealed a significantly increased (-)675-nm polymer- or salt-induced (psi)-type $\mathrm{CD}$ in $v c c n 1-1$ as compared with the wt (Supplementary Fig. 21), indicating tighter stacking of grana ${ }^{31}$ in the mutant, in agreement with SANS data. At the same time, $v c c n 1-1$, similar to the wt, exhibited intense psi-type bands at around $(+) 505$ and $(+) 690 \mathrm{~nm}$, arising from the long-range chiral order of LHCII-PSII super-complexes ${ }^{32}$, suggesting that the overall macro-organization of pigment-protein complexes in grana was not perturbed by the mutation (Supplementary Fig. 21). Taken together, these data suggest the influence of AtVCCN1 on thylakoid ultrastructure in addition to its role in photosynthetic regulation.

\section{Discussion}

Experimental evidence has recently shown that $\mathrm{K}^{+}$fluxes by AtTPK3 and AtKEA3 in thylakoids regulate photosynthesis by modulation of PMF composition ${ }^{3,11,12}$. Activities of $\mathrm{Cl}^{-}$channels have been reported previously in thylakoids and hypothesized to play role in charge counterbalance of $\mathrm{H}^{+}$ pumping ${ }^{6,7}$, and a member of the ClC chloride channel family, $\mathrm{ClCe}$, has been located to thylakoids ${ }^{14-16}$. However, there is no experimental evidence for AtClCe being a $\mathrm{Cl}^{-}$channel. Therefore, it can neither be excluded nor confirmed that $\mathrm{ClCe}$ contributes to the previously reported activities. In this study, we bring evidence that AtVCCN1 forms a voltage-dependent $\mathrm{Cl}^{-}$ channel, is located in the thylakoid membrane, and displays electrophysiological characteristics similar to those of the elusive activity previously reported. AtVCCN1 has homologues in plants, bacteria, algae, fungi and arthropods, suggesting that its function in $\mathrm{Cl}^{-}$flux and membrane potential dissipation may be phylogenetically conserved, and have a widespread importance 


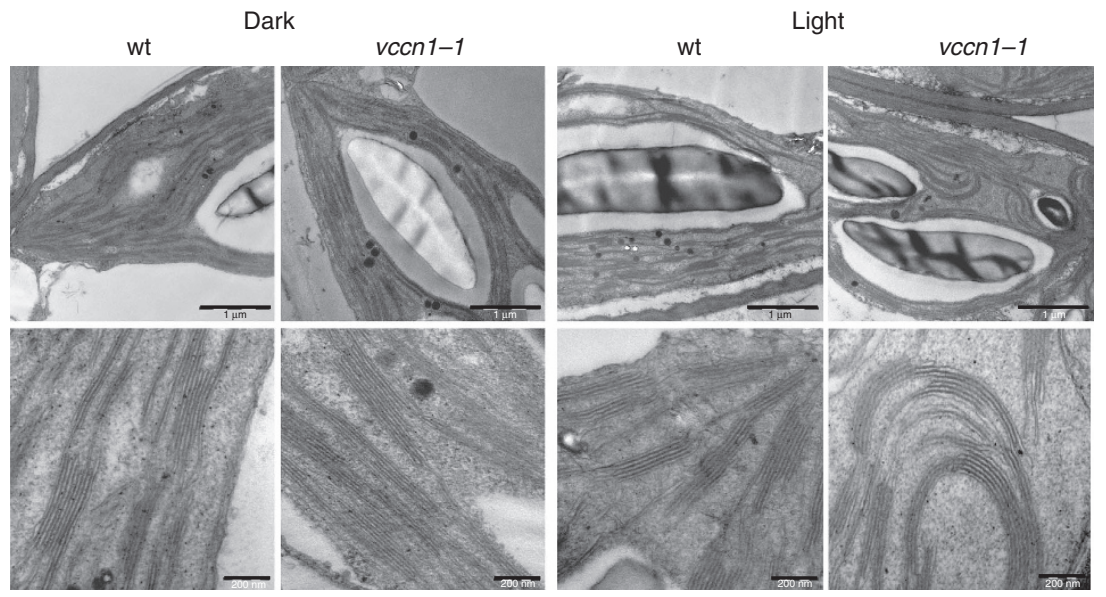

Figure 5 | Thylakoid ultrastructure. Representative transmission electron micrographs are shown for leaf chloroplasts from 8-week-old wild-type (wt) plants and vccn $1-1$ mutant fixed after $16 \mathrm{~h}$ of darkness or $3 \mathrm{~h}$ after the onset of illumination ( $150 \mu \mathrm{mol}$ photons per $\mathrm{m}^{2}$ per $\mathrm{s}$ ). The mutant shows consistently longer thylakoid stacks (grana) than wt in the dark state. In light conditions, the grana of vccn1-1 are also longer, but often display a curved 'banana-like' shape (observed in $20-50 \%$ of the studied plastid sections). Scale bars, $1 \mu \mathrm{m}$ (upper panels) and $200 \mathrm{~nm}$ (lower panels).

to cope with rapid changes in natural habitats. AtVCCN2 is a close paralogue, but is not functionally redundant to AtVCCN1 in leaves, where its expression is low.

Using electrophysiology in planar lipid bilayers we show that AtVCCN1 mediates flux of $\mathrm{Cl}^{-}$in response to the applied voltage (Fig. 2, Supplementary Figs 6,7,9,10). In wt thylakoid membranes, the $\Delta \Psi$ component of PMF is the voltage that activates and regulates AtVCCN1 to transport $\mathrm{Cl}^{-}$into the lumen, which results in partial dissipation of $\Delta \Psi$ (Fig. 3). A significant $\Delta \Psi$ persists across wt thylakoids under steady-state conditions, in agreement with previous work by Kramer et al. ${ }^{8}$ Our observations appear to differ from those in the recent report by Johnson and Ruban ${ }^{33}$, who suggested that $\Delta \Psi$ is completely and rapidly dissipated by ion fluxes during illumination. In our conditions, complete dissipation of $\Delta \Psi$ was observed only in oeVCCN1, most likely due to an elevated accumulation of negative charges $\left(\mathrm{Cl}^{-}\right)$in the lumen of this genotype.

Through partial dissipation of $\Delta \Psi$, AtVCCN1 could also reduce the size of total PMF. Thus AtVCCN1 could be one of the factors determining the PMF size, together with the photosynthetic linear and cyclic electron transport and the $\mathrm{H}^{+}$ efflux through ATP synthase ${ }^{8,34}$. Overexpression of AtVCCN1 led to increased rate of $\mathrm{H}^{+}$efflux through ATP synthase despite a lower PMF than in wt, without impacting plant growth under standard cultivation conditions (Fig. 3, Supplementary Fig. 11, Supplementary Table 1). Together with similar observations by Wang et al. ${ }^{34}$ for mutants affected in cyclic electron transport, our data suggest that even a lower PMF size could be sufficient for the activity of the ATP synthase during steady-state photosynthesis and plant growth. Overexpression of AtVCCN1 resulted in PMF consisting solely of $\Delta \mathrm{pH}$ (Fig. 3), and an enhanced acidification of the thylakoid lumen (Supplementary Fig. 14). These observations are in line with the higher NPQ and lower electron transport (Fig. 3, Supplementary Fig. 19). On the basis of our results, we propose that the lower lumenal $\mathrm{pH}$ did not limit but rather stimulated the activity of the ATP synthase.

The wt-like phenotype of $v c c n 2$ mutants in all studied PMF and photosynthesis parameters (Supplementary Figs 17,18) could be explained by a possible compensation by AtVCCN1 for the function of AtVCCN2. However, the double vccn1vccn 2 mutant did not display an enhanced phenotype but resembled $v c c n 1$. These findings together with the limited expression of AtVCCN2 in leaves make it unlikely that it is functionally redundant to AtVCCN1 in the regulation of PMF and photosynthesis in leaf thylakoids. The possibility that AtVCCN2 could form an anion channel as well, based on the close homology to AtVCCN1 and similar effects on PMF caused by its overexpression, requires further investigations.

Participation of AtVCCN1 in the regulation of PMF and photosynthesis ascribes this protein a function in the rapid acclimation of plants in variable light environments (Fig. 4). We propose that AtVCCN1 plays distinct roles as well as roles in common with AtTPK3 and AtKEA3 in photosynthetic acclimation. Like these $\mathrm{K}^{+}$transport systems, AtVCCN1 modulates PMF partitioning into $\Delta \mathrm{pH}$ and $\Delta \Psi$, affecting the balance between electron transport and photoprotective mechanisms. Distinctly, AtVCCN1 is activated immediately on the transition from dark to light by the establishment of $\Delta \Psi$, and is thus an early component modulating the PMF. Activation of AtTPK3 and AtKEA3 may require a significant $\Delta \mathrm{pH}$ to be established by the partial dissipation of $\Delta \Psi$, for example by AtVCCN1. Furthermore, AtVCCN1 acts in photosynthetic acclimation after transitions to high light, whereas AtKEA3 activity is important for transitions to low light. Finally and distinctly from AtTPK3 and AtKEA3, AtVCCN1 alters the total PMF size and activity of ATP synthase, which may be a strategy to adjust photosynthetic carbon fixation in addition to electron transport. Our findings make AtVCCN1 an early component acting to rapidly adjust plant photosynthesis in variable light environments, and therefore it may be a target for improving photosynthetic acclimation in fields for agricultural and bioenergy production.

\section{Methods}

Plant material and growth conditions. Arabidopsis thaliana wt plants (Columbia ecotype) and mutants were grown in soil for 7-8 weeks in a growth chamber (CLF PlantMaster; Plant Climatics, Wertingen, Germany) using a daily cycle of $8 \mathrm{~h}$ of light $\left(150 \mu \mathrm{mol}\right.$ photons per $\mathrm{m}^{2}$ per s) at $20^{\circ} \mathrm{C}$ and $16 \mathrm{~h}$ of dark at $19^{\circ} \mathrm{C}$ at relative humidity of $60 \%$. The $v c c n 1-1$ (SALK_103612) and $v c c n 2-1$ (SALK_114715) mutants were obtained from the SALK collection $^{35}$, vccn1-2 (GABI_796C09) from the GABI-KAT collection ${ }^{36}$, and $v c c n 2-2$ (SK_2655) from the Saskatoon collection $^{37}$. The double $v c c n 1 v c c n 2$ mutant was obtained by crossing $v c c n 1-1$ and $v c c n 2-1$. Reverse transcription-PCR with gene-specific primers was used to confirm the absence of transcript in the corresponding mutants (Supplementary Fig. 11b, Supplementary Table 3). ACTIN8 (At1g49240) was used as the reference gene.

AtVCCN-GFP fusions were constructed as follows. The complete coding sequence of AtVCCN1 or AtVCCN2 (excluding native stop codons), were synthesized with flanking att $\mathrm{L} 1$ and $a t t \mathrm{~L} 2$ sites and cloned into vector pMK-RQ (GeneArt, Life Technologies, Carlsbad, CA, USA). Gene fragments were transferred to the binary vector pB7FWG2 (ref. 38) containing the CaMV 35S promoter and eGFP at the C-termini using Gateway recombinational cloning 
(Invitrogen, Life Technologies). Constructs were transformed into the Agrobacterium tumefaciens strain GV3101 and subsequently in Arabidopsis vccn1-1 or $v c c n 2-1$ genetic backgrounds, respectively, using the floral dip method ${ }^{39}$. To select for successfully transformed plants, seeds were sown on soil and sprayed for 3 days at the fourth leaf stage with $60 \mathrm{mg}$ per ml glufosinate (Basta; Hoechst Schering AgrEvo, Düsseldorf, Germany). GFP expression in transformed plants was verified using an Axio Scope Al epifluorescence microscope (Carl Zeiss Microscopy, Göttingen, Germany). Presence of the VCCN transgene in transformants was confirmed by PCR and its expression verified by quantitative PCR.

Quantitative PCR. Total RNA was isolated from plant tissues of 7-week-old plants with an E.Z.N.A. R6827-01 Plant RNA kit (Omega Bio-Tek, Norcross, GA, USA) and residual DNA was removed with E1091 DNAse (Omega Bio-Tek). Tissues pooled from 30 plants were used for each analysis. Complementary DNA (cDNA) was prepared from $400 \mathrm{ng}$ RNA with an iScript cDNA Synthesis kit (Bio-Rad, Hercules, CA, USA). Quantitative real-time PCR analyses were conducted with a SsoAdvanced Universal SYBR Green Supermix on a C1000 Touch Thermal Cycler (Bio-Rad). PCR template cDNA corresponding to $40 \mathrm{ng}$ total RNA was used in $15-\mu \mathrm{l}$ reactions. Amplifications were undertaken in two-step PCR with the following cycling conditions: initial denaturation for $3 \mathrm{~min}$ at $95^{\circ} \mathrm{C}$, followed by 40 cycles of denaturation for $5 \mathrm{~s}$ at $95^{\circ} \mathrm{C}$ and annealing/extension for $30 \mathrm{~s}$ at $60^{\circ} \mathrm{C}$. Melt-curve analyses were conducted for all primers after amplification. Expected product sizes were confirmed by agarose gel electrophoresis. Efficiencies of PCR amplification of all target genes were established from serially diluted calibration curves of wt cDNA prepared from leaf tissue. Gene-specific primers used are listed in Supplementary Table 3. Relative expression was calculated with the $\Delta$ Cq method $\left(2^{-\Delta \mathrm{Cq}}\right)$ using PEX4 (At5g25760) as the reference gene.

\section{Subcellular localization of AtVCCN-GFP fusion proteins. Protoplasts were} prepared using the Tape-Arabidopsis Sandwich procedure from leaves of transgenic plants (T2 generation) expressing AtVCCN-GFP $P^{40}$. Protoplasts were mounted in W1 solution ( $0.5 \mathrm{M}$ mannitol, $20 \mathrm{mM} \mathrm{KCl}, 4 \mathrm{mM} \mathrm{MES-KOH,} \mathrm{pH} \mathrm{5.7)}$ and observed using a LSM 700 inverted Axio Observer Z1 confocal laser scanning microscope (Carl Zeiss Microscopy, Jena, Germany) equipped with a LD C-Apochromat water immersion objective lens ( $\times$ 40/1.1 NA). GFP and chlorophyll fluorescence were both excited at $488 \mathrm{~nm}$ and emitted signals were collected above $640 \mathrm{~nm}$ for chlorophyll fluorescence (long-pass filter) and below $548 \mathrm{~nm}$ for GFP fluorescence (variable secondary dichroic beamsplitter). Images were analysed with Zen 2012 blue edition software (Carl Zeiss Microscopy).

Electrophysiology measurements. The AtVCCN1 gene was PCR-amplified without the targeting peptide (corresponding to the first 40 amino acids) from Arabidopsis leaf cDNA introducing the restriction sites NdeI $\left(5^{\prime}\right)$ and SacI $\left(3^{\prime}\right)$ and cloned into the pIVEX1.4 WG vector (Roche, Basel, Switzerland) in fusion with a $6 \times$ His-tag. Gene-specific primers used are listed in Supplementary Table 3. In vitro expression was conducted using a RTS 100 Wheat Germ CECF kit (Roche Diagnostics, Basel, Switzerland) according to manufacturer's instructions. The expressed protein present in the lysate, which does not contain other membrane proteins, was solubilized with $2 \%(\mathrm{w} / \mathrm{v})$ Triton X-100.

Experiments involving planar lipid bilayers were carried out as described previously ${ }^{11}$. Briefly, a BC-525C electrophysiological planar bilayer apparatus (Warner Instruments, Hamden, CT, USA) was used. Bilayers with a capacity of $\sim 150-200 \mathrm{pF}$ were prepared by painting a decane/chloroform solution of soybean asolectin (Sigma-Aldrich, St Louis, MO, USA), purified by precipitation with cold acetone from a chloroform solution, across a $250-\mu \mathrm{m}$ hole in a polystyrene cuvette. Solutions consisting of $100 \mathrm{mM} \mathrm{KCl}$ or $100 \mathrm{mM} \mathrm{KNO}_{3}$ in $10 \mathrm{mM}$ Hepes- $\mathrm{KOH} \mathrm{pH}$ 7.0 were used. For ionic gradient experiments, $300 \mathrm{mM}$ and $100 \mathrm{mM} \mathrm{KCl}$ in the same buffer were used on the cis and trans side, respectively. Contents of both chambers were stirred by magnetic bars when necessary. Connections to electrodes were provided by agar bridges. The protein expressed in wheat germ lysate was added to the cis side. Addition of empty wheat germ lysate never resulted in channel activity (Supplementary Fig. 5b). Voltages reported are those of the cis chamber, and current is considered to be positive when carried by cations flowing from the cis compartment to the trans compartment. The recorded data were analysed using the pCLAMP8.0 program set (Molecular Devices, Sunnyvale, CA, USA).

Protein and pigment analyses. For localization studies, chloroplasts were isolated from leaves of dark-adapted plants, which were grinded in preparation media (50 mM Hepes-KOH pH 7.8, $330 \mathrm{mM}$ Sorbitol, $10 \mathrm{mM} \mathrm{KCl}$ and $1 \mathrm{mM} \mathrm{EDTA}$ ), filtrated through two layers of Miracloth and subsequently centrifuged at $3,000 \mathrm{~g}$ for $3 \mathrm{~min}$ at $4^{\circ} \mathrm{C}$. The pellet representing the crude chloroplast fraction was resuspended in preparation buffer, loaded and centrifuged in a 10\% (v/v) Percoll solution at $3,000 \mathrm{~g}$ for $10 \mathrm{~min}$ (ref. 41). After lysis of purified chloroplasts, thylakoids and envelope were purified on a four-step sucrose gradient by centrifugation at $70,000 \mathrm{~g}$ for $1 \mathrm{~h}$ at $4{ }^{\circ} \mathrm{C}$ (ref. 42). Thylakoid subfractions, grana and stroma lamellae, were isolated by solubilization of thylakoids with digitonin ${ }^{43}$. Thylakoids at $0.5 \mathrm{mg}$ chlorophyll per $\mathrm{ml}$ in $25 \mathrm{mM}$ Tricine- $\mathrm{KOH} \mathrm{pH} 7.8,100 \mathrm{mM}$ Sorbitol, $5 \mathrm{mM} \mathrm{MgCl}_{2}, 10 \mathrm{mM} \mathrm{KCl}$ and $10 \mathrm{mM} \mathrm{NaF}$ were solubilized with $1 \%(\mathrm{w} / \mathrm{v})$ digitonin by gentle stirring for $7 \mathrm{~min}$ at room temperature. The suspension was centrifuged at $1,000 \mathrm{~g}$ for $5 \mathrm{~min}$ to remove insolubilized material. The supernatant was further centrifuged at $40,000 \mathrm{~g}$ for $30 \mathrm{~min}$ and at $140,000 \mathrm{~g}$ for $90 \mathrm{~min}$, resulting in the grana and the stroma lamellae fractions. Chlorophyll concentration of various fractions was determined following extraction in $80 \%(\mathrm{v} / \mathrm{v})$ acetone for $5 \mathrm{~min}$ at $4{ }^{\circ} \mathrm{C}$ and spectrophotometry ${ }^{44}$.

SDS/urea/PAGE and immunoblotting using antibodies against various proteins were conducted as described by Yin et al. ${ }^{45}$ Antibodies against PsbS (AS09533, 1/2,000), Lhcb2 (AS01003, 1/20,000), Cytf (AS08306, 1/2,000), PsaA (AS06172, 1/1,000), PsaB (AS10695, 1/1,000), AtpB (AS05085, 1/1,000), Toc34 (AS07238, 1/1,000), and RbcL (AS03037, 1/10,000) were purchased from Agrisera (Vännäs, Sweden). The antibodies against D2 and CP43 proteins from spinach $(1 / 5,000)$ were kindly provided by Eva-Mari Aro (Turku University) and Torill Hundal (Stockholm University). Anti-GFP antibody (Cat\#11814460001, 1/5,000) was obtained from Roche Diagnostics (Indianapolis, IN, USA). Immunodetection was done using an ECL system WesternBright Quantum (Science Imaging Scandinavia, Stockholm, Sweden) and visualized using Fusion SL7 (Vilbert Lourmat, Eberhardzell, Germany). Uncropped versions of the immunoblots from Fig. 1 and Supplementary Fig. 12 are shown in Supplementary Figs 4 and 22 , respectively.

Chlorophyll and carotenoid contents of leaf discs were determined following extraction in $96 \%$ (v/v) ethanol for $10 \mathrm{~min}$ at $60^{\circ} \mathrm{C}$ and spectrophotometry ${ }^{46}$.

Measurements of chlorophyll a fluorescence. Slow kinetics of chlorophyll fluorescence induction were recorded with a pulse-amplitude modulated fluorometer DUAL-PAM 100 (Walz, Effeltrich, Germany) on attached leaves of 30-min dark-adapted plants at room temperature at actinic red light of given intensity. NPQ and $\Phi_{\mathrm{II}}$ were calculated as $\left(F_{\mathrm{m}}-F_{\mathrm{m}}{ }^{\prime}\right) / F_{\mathrm{m}}{ }^{\prime}$ and $\left(F_{\mathrm{m}}{ }^{\prime}-F\right) / F_{\mathrm{m}}{ }^{\prime}$, respectively ${ }^{47}$, where $F_{\mathrm{m}}$ is maximum fluorescence yield, $F_{\mathrm{m}}{ }^{\prime}$ is maximum fluorescence yield in the light, and $F$ is variable fluorescence yield in the light.

Electrochromic band shift measurements. ECS was recorded with a DUAL-PAM 100 system (Walz) equipped with a P515/535 emitter/detector module as absorbance difference signal $550-515 \mathrm{~nm}$ according to Walz protocols ${ }^{48}$. First, plants were dark adapted for $30 \mathrm{~min}$, and then exposed to actinic red light of given intensity for $\leq 10 \mathrm{~min}$. The light was turned off and the ECS decay kinetics were recorded to determine total ECS $\left(\right.$ ECS $\left._{\mathrm{t}}\right)$ representing total PMF size and its components $(\Delta \mathrm{pH}$ and $\Delta \Psi)$ according to the method of Cruz and colleagues ${ }^{49}$. Before each measurement, three pulses of $5-\mu$ s and $200,000 \mu$ mol photons per $\mathrm{m}^{2}$ per $\mathrm{s}$ were applied and the signals were averaged to determine $\mathrm{ECS}_{\mathrm{ST}}$, which was used to normalize the ECS $\mathrm{t}_{\mathrm{t}}$ values of each measurement. Normalization was done by multiplying $\mathrm{ECS}_{\mathrm{t}}$ with a correction factor calculated as $\left(\operatorname{maxECS}_{\mathrm{ST}} / E C S_{S T}\right)$, where maxECS $\mathrm{ST}_{\mathrm{ST}}$ is the highest $\mathrm{ECS}_{\mathrm{ST}}$ from all the measurements of a dataset, and $E C S_{S T}$ is the ECS $\mathrm{ST}_{\mathrm{ST}}$ corresponding to the measurement of the $\mathrm{ECS}_{\mathrm{t}}$ that is being normalized. To determine $\mathrm{H}^{+}$conductivity of the thylakoid membrane through ATP synthase $\left(\mathrm{g}_{\mathrm{H}}^{+}\right)$, the light was turned off at specific time periods and the decay of the ECS signal was recorded during $600 \mathrm{~ms}$ dark intervals. The ECS decay was fitted with a single exponential function for the first $100 \mathrm{~ms}$, and $\mathrm{g}_{\mathrm{H}}^{+}$was calculated as $1 /$ time constant for deca ${ }^{50}$. Where indicated, leaf discs were incubated for $10 \mathrm{~min}$ in a solution containing $1 \%(\mathrm{v} / \mathrm{v})$ Tween $20,2 \%(\mathrm{v} / \mathrm{v})$ DMSO and $5 \mathrm{mM}$ DCCD before illumination and ECS recording.

Measurement of $\mathbf{p H}$ changes by $\mathbf{A O}$ fluorescence. The fluorescent probe $\mathrm{AO}$ was used to measure thylakoid membrane energization ${ }^{51,52}$. Although a simple relation between fluorescence quenching attributable to accumulation of protonated amines in the lumen and $\mathrm{pH}$ has been proposed, this method only allows a semiquantitative indication of $\mathrm{pH}$ changes ${ }^{51,52}$. Intact chloroplasts were isolated from Arabidopsis leaves and purified by a two-step Percoll gradient centrifugation ${ }^{53}$ Time-resolved measurements of AO using Arabidopsis chloroplasts were performed with a PAM101/102/103 fluorometer (Walz, Effeltrich, Germany) endowed with a GFP filter set (excitation at $480 \mathrm{~nm}$; cutoff, $<505 \mathrm{~nm}$; emission with long-pass filter, $515 \mathrm{~nm}$; cutoff, $575 \mathrm{~nm}$ ). Actinic light was applied by fibre optic and was low enough to avoid actinic effects. AO fluorescence was measured in darkness and during illumination for $100 \mathrm{~s}$ at $700 \mu \mathrm{mol}$ photons per $\mathrm{m}^{2}$ per $\mathrm{s}$. Chlorophyll concentration was adjusted to $10 \mu \mathrm{g}$ per $\mathrm{ml}$ in the purification resuspension buffer $(20 \mathrm{mM}$ Hepes-KOH pH 7.6, 0.4 M Sorbitol, $2.5 \mathrm{mM}$ EDTA, $5 \mathrm{mM} \mathrm{MgCl}_{2}, 10 \mathrm{mM} \mathrm{NaHCO}_{3}$, $0.15 \%(\mathrm{w} / \mathrm{v}) \mathrm{BSA})$ and the chloroplast suspension was preincubated with $1 \mu \mathrm{M} \mathrm{AO}$ for $20 \mathrm{~min}$ in darkness before measurement.

Transmission electron microscopy. Sections $(\sim 1 \mathrm{~mm} \times 1 \mathrm{~mm})$ cut from the central parts of leaf blades of 8 -week-old plants were fixed in $2.5 \%(\mathrm{v} / \mathrm{v})$ glutaraldehyde for 4 days, then post-fixed in $1 \% \mathrm{OsO}_{4}(\mathrm{w} / \mathrm{v})$ for $2 \mathrm{~h}$. Fixatives were buffered with $70 \mathrm{mM} \mathrm{Na} 2 \mathrm{HPO}_{4}-\mathrm{KH}_{2} \mathrm{PO}_{4} \mathrm{pH}$ 7.2. After fixation steps, samples were rinsed in the same buffer. After dehydration in an alcohol series, samples were embedded in Durcupan ACM resin (Fluka, Buchs, Switzerland). Ultrathin sections (thickness 50-60 nm) were cut with an UC6 ultramicrotome (Leica Microsystems, Vienna, Austria) fitted with a diamond knife (Diatome, Hatfield PA, USA). Sections were mounted on copper grids and contrasted with 5\% uranyl acetate and Reynolds' lead citrate solution. TEM was conducted with a LEO 912AB Gemeni 
system (Carl Zeiss, Oberkochen, Germany) equipped with a LaB6 emitter at an accelerating voltage of $120 \mathrm{kV}$ using a Veleta CCD camera and iTEM software (Olympus Soft Imaging Systems, Münster, Germany).

ImageJ software was used to measure granum diameter (measured at the middle of perpendicular granum sections) on transmission electron micrographs. Calculations were done on randomly chosen grana (350-450) originating from 60-90 different plastids taken randomly from 60-90 different mesophyll cells from leaves of two different plants per treatment. Statistical analyses were undertaken using InStat v.6.0 (GraphPad, La Jolla, CA, USA). Ultrastructural data did not follow a normal distribution, so Kruskal-Wallis non-parametric ANOVA was used followed by the Mann-Whitney rank-sum test. For all data, $P<0.05$ was considered significant.

SANS measurements. SANS measurements were carried out on the SANS-I (leaves) and SANS-II instruments (isolated thylakoid membranes) at the Paul Scherrer Institute (Villigen, Switzerland). On SANS-I and SANS-II, we used a wavelength of $6 \AA$, collimation of 11 and $6 \mathrm{~m}$, and sample-detector distance of 11 and $6 \mathrm{~m}$, respectively. Detached leaves were infiltrated in $\mathrm{D}_{2} \mathrm{O}$ to enhance contrast. Owing to the relatively weak Bragg peak in Arabidopsis leaves compared with several other species ${ }^{30}$, for each measurement 8-10 leaves were placed in a quartz cuvette (path length, $2 \mathrm{~mm}$ ) filled with $\mathrm{D}_{2} \mathrm{O}$. To improve the signal-to-noise ratio, experiments were repeated three times for $v c c n 1-1$ and four times for $\mathrm{wt}$, and the SANS curves were averaged. Hence, these SANS profiles represent the statistical average of $\sim 25-30$ leaves. Thylakoid membranes, isolated as described by Posselt and colleagues ${ }^{54}$, were suspended in a $\mathrm{D}_{2} \mathrm{O}$-containing reaction medium $(20 \mathrm{mM}$ Tricine, pD 7.6, $0.4 \mathrm{M}$ sorbitol, $5 \mathrm{mM} \mathrm{MgCl} 2,5 \mathrm{mM} \mathrm{KCl}$ ) and measured at a chlorophyll concentration of $\sim 500 \mu \mathrm{g}$ per ml in a quartz cuvette (optical path length $2 \mathrm{~mm}$ ) placed in a magnetic field of $0.4 \mathrm{~T}$.

Primary steps of data analyses and radial averaging in a $360^{\circ}$ sector (leaves) and in two $75^{\circ}$ sectors (isolated thylakoid membrane) of the two-dimensional scattering profiles were performed with the Graphical Reduction and Analysis SANS Program package (GRASP) developed by C. Dewhurst (Institut Laue-Langevin, Grenoble, France). $\mathrm{RD}$ of the thylakoid membrane was calculated from the position of the characteristic Bragg peak, which corresponded to the periodicity of grana thylakoid membranes. For this, SANS curves in $0.0151-0.0380 \AA^{-1}$ (leaves) and $0.0194-$ $0.0310 \AA^{-1}$ (isolated thylakoids) regions were fitted with equation (1):

$$
I(q)=I_{0}+A *|q|^{\mathrm{p}}+B * \mathrm{e}^{-\frac{\left(q-q^{*}\right)^{2}}{2 c^{2}}},
$$

where $I$ is the scattered intensity, $I_{0}, A$ and $B$ are constants, $q$ is scattering vector, $q^{*}$ is position of the Bragg peak, $p$ and $c$ are parameters of the power function and Gaussian distribution, respectively ${ }^{55}$. The $q^{*}$ parameter was used to calculate RD with equation (2):

$$
\mathrm{RD}=2 \pi / q^{*}
$$

For detached leaves, statistically averaged RD values for 25-30 leaves per treatment are given; the corresponding error values arise from uncertainty of the fitting, containing the statistical error of the scattering intensity data points. SANS profiles of untreated and salt-treated thylakoids were measured on freshly isolated membranes; the variation of $\mathrm{RD}$ values among repeated measurements within $3 \mathrm{~h}$ was $<1 \AA$. Representative one-dimensional plots and RD values with the error of fitting are presented.

CD spectroscopy. CD measurements were made on a J-815 spectropolarimeter (JASCO, Tokyo, Japan). Detached, water-infiltrated leaves were placed between two glass slides in an optical cell. Spectra were recorded at room temperature between 400 and $800 \mathrm{~nm}$ at a scan speed of $100 \mathrm{~nm}$ per min, band-pass of $3 \mathrm{~nm}$ and step size of $1 \mathrm{~nm}$. For each sample, 3-4 scans were averaged. Spectra were normalized to the absorption of the red-most peak of the spectra recorded at the same time as the CD spectra and were corrected for baseline distortions. Measurements were repeated on three different leaves for each genotype. Amplitudes of psi-type CD bands, at around $(+) 505,(-) 675$ and $(+) 690 \mathrm{~nm}$, were determined using the reference wavelengths of 550,600 and $750 \mathrm{~nm}$, respectively.

Phylogenetic analyses. To identify candidates for the most closely related eukaryotic protein sequences to AtVCCN1, we used BlastP searches with 'AtVCCN1' as the query against the GenBank database ${ }^{56}$. Initially, we selected best matches from representative green plant lineages to build up the probable orthologous group of sequences from these lineages. Sampled lineages included the land-plant families Brassicaceae, Fabaceae, Solanaceae, Vitaceae, Poaceae, Pinaceae, Selaginellaceae, Funariaceae, and the green alga families Chlorellaceae and Chlamydomonadaceae.

Non-green-plant best matches were very few and had low $E$ values. Among the best matches were representatives of haptophytes (Emeliana $E=7 \times 10^{-40}$ ), heterokontophytes (Phaeodactylum $E=5 \times 10^{-37}$ ) and cyanobacteria (Synechococcus $E=9 \times 10^{-28}$ ), with $16-25 \%$ maximum identity to AtVCCN1 (based on MUSCLE alignment). Specific searches to bacteria also retrieved the same cyanobacterium best match as observed previously. We included representative matches of each of these lineages in our alignments (Supplementary Data 1).
Specific searches for opisthokonta targets (the clade including animals and fungi) yielded matches with $E$ values $\leq 10^{-15}$, with matches between $10^{-15}$ and $10^{-11}$ (the top 10 matches) all from fungi, with $12-14 \%$ maximum identity to AtVCCN1 (Supplementary Data 1). Representative fungal sequences as well as BEST1 from human were included to evaluate the type of homology present between AtVCCN1 and BEST1 (that is, if they are orthologous, paralogous, or not even homologous).

Sequences were aligned using automated methods: MAFFT, MUSCLE, T-COFFEE and CLUSTAL-O (default values in each case, using the EMBL server at www.ebi.ac.uk/Tools/msa/). BEST1 and AtVCCN1 sequences were compared using a BLOSUM62 matrix. Then, preliminary neighbour-joining (NJ) analyses were conducted on alignments. When including BEST1 from humans, only $9 \%$ identity was found with AtVCCN1 (MUSCLE alignment in

Supplementary Fig. 1), and so this sequence was excluded from the main phylogenetic analyses. Neighbour-joining trees were used to assess which branches in the phylogeny were particularly long in comparison with others in the tree. Further searches in BlastP were conducted using sequences on long branches to sample additional closely related sequences. These were added to the tree so that these long branches would be subdivided, thereby making later phylogenetic estimates likely to be more robust ${ }^{57}$. The best BlastP match to red algae (Galdieria, $E=5 \times 10^{-22}, 32 \%$ maximum identity) was also included because this group can help to understand the host versus the symbiont origin of genes ${ }^{23}$

The sequence from all taxa corresponding to the chloroplast-targeting region in AtVCCN1 (residues 1-40) and of the LALD amino-acid motif common to most land plants, were trimmed before running the main analyses. These analyses were conducted using the Bayesian inference in MrBayes 3.2.2 (ref. 58): (a) using the same trimmed MAFFT alignment as described previously for all taxa except human BEST1 (confirmed using PhyML ${ }^{59}$ ), and (b) using the corresponding DNA sequences from the genes found only in Brassicaceae and Vitaceae, with the addition of a Cleomaceae sequence (sister family to Brassicaceae ${ }^{60}$ ), to clarify when the gene duplication that produced VCCN1 and VCCN2 occurred. MrBayes settings included reversible model jump MCMC over the substitution models, four chains, and paired runs for 5 million generations.

Data availability. The data that support the findings of this study are available from the corresponding authors on request.

\section{References}

1. Finazzi, G. et al. Ions channels/transporters and chloroplast regulation. Cell Calcium 58, 86-97 (2014).

2. Pribil, M., Labs, M. \& Leister, D. Structure and dynamics of thylakoids in land plants. J. Exp. Bot. 65, 1955-1972 (2014)

3. Armbruster, U. et al. Ion antiport accelerates photosynthetic acclimation in fluctuating light environments. Nat. Commun. 5, 5439 (2014).

4. Hind, G., Nakatani, H. Y. \& Izawa, S. Light-dependent redistribution of ions in suspensions of chloroplast thylakoid membranes. Proc. Natl Acad. Sci. USA 71, 1484-1488 (1974).

5. Segalla, A., Szabó, I., Costantini, P. \& Giacometti, G. M. Study of the effect of ion channel modulators on photosynthetic oxygen evolution. J. Chem. Inf. Model 45, 1691-1700 (2005).

6. Enz, C., Steinkamp, T. \& Wagner, R. Ion channels in the thylakoid membrane (a patch-clamp study). Biochim. Biophys. Acta 1143, 67-76 (1993).

7. Schönknecht, G., Hedrich, R., Junge, W. \& Raschke, K. A voltage-dependent chloride channel in the photosynthetic membrane of a higher plant. Nature 336, 589-592 (1988).

8. Kramer, D. M., Cruz, J. A. \& Kanazawa, A. Balancing the central roles of the thylakoid proton gradient. Trends Plant Sci. 8, 27-32 (2003).

9. Avenson, T. J., Cruz, J. A., Kanazawa, A. \& Kramer, D. M. Regulating the proton budget of higher plant photosynthesis. Proc. Natl Acad. Sci. USA 102, 9709-9713 (2005).

10. Murchie, E. H. \& Niyogi, K. K. Manipulation of photoprotection to improve plant photosynthesis. Plant Physiol. 155, 86-92 (2011).

11. Carraretto, L. et al. A thylakoid-located two-pore $\mathrm{K}(+)$ channel controls photosynthetic light utilization in plants. Science 342, 114-118 (2013).

12. Kunz, H. H. et al. Plastidial transporters KEA1, -2 , and -3 are essential for chloroplast osmoregulation, integrity, and $\mathrm{pH}$ regulation in Arabidopsis. Proc. Natl Acad. Sci. USA 111, 7480-7485 (2014).

13. Pottosin, I. I. \& Schönknecht, G. Patch clamp study of the voltage-dependent anion channel in the thylakoid membrane. J. Membr. Biol. 148, 143-156 (1995).

14. Teardo, E. et al. Localization of a putative ClC chloride channel in spinach chloroplasts. FEBS Lett. 579, 4991-4996 (2005).

15. Marmagne, A. et al. Two members of the Arabidopsis CLC (chloride channel) family, AtCLCe and AtCLCf, are associated with thylakoid and Golgi membranes, respectively. J. Exp. Bot. 58, 3385-3393 (2007).

16. Lv, Q. D. et al. Cloning and molecular analyses of the Arabidopsis thaliana chloride channel gene family. Plant Sci. 176, 650-661 (2009).

17. Monachello, D. et al. Two anion transporters AtClCa and AtClCe fulfil interconnecting but not redundant roles in nitrate assimilation pathways. New Phytol. 183, 88-94 (2009). 
18. Ajjawi, I., Lu, Y., Savage, L. J., Bell, S. M. \& Last, R. L. Large-scale reverse genetics in Arabidopsis: case studies from the Chloroplast 2010 Project. Plant Physiol. 152, 529-540 (2010).

19. Fristedt, R. et al. PHOTOSYSTEM II PROTEIN33, a protein conserved in the plastid lineage, is associated with the chloroplast thylakoid membrane and provides stability to photosystem II supercomplexes in Arabidopsis. Plant Physiol. 167, 481-492 (2015).

20. Lu, Y., Hall, D. A. \& Last, R. L. A small zinc finger thylakoid protein plays a role in maintenance of photosystem II in Arabidopsis thaliana. Plant Cell 23, 1861-1875 (2011).

21. Kane Dickson, V., Pedi, L. \& Long, S. B. Structure and insights into the function of a $\mathrm{Ca}(2+)$-activated $\mathrm{Cl}(-)$ channel. Nature 516, 213-218 (2014).

22. Schwacke, R. et al. ARAMEMNON, a novel database for Arabidopsis integral membrane proteins. Plant Physiol. 131, 16-26 (2003).

23. Pfeil, B. E., Schoefs, B. \& Spetea, C. Function and evolution of channels and transporters in photosynthetic membranes. Cell. Mol. Life Sci. 71, 979-998 (2014).

24. Zimmermann, P., Hennig, L. \& Gruissem, W. Gene-expression analysis and network discovery using Genevestigator. Trends Plant Sci. 10, 407-409 (2005).

25. Berrier, C. et al. Cell-free synthesis of a functional ion channel in the absence of a membrane and in the presence of detergent. Biochemistry 43, 12585-12591 (2004).

26. Deniaud, A., Liguori, L., Blesneac, I., Lenormand, J. L. \& Pebay-Peyroula, E. Crystallization of the membrane protein hVDAC1 produced in cell-free system. Biochim. Biophys. Acta 1798, 1540-1546 (2010).

27. De Stefani, D., Raffaello, A., Teardo, E., Szabó, I. \& Rizzuto, R. A fortykilodalton protein of the inner membrane is the mitochondrial calcium uniporter. Nature 476, 336-340 (2011).

28. Hille, B. Ion Channels of Excitable Membranes 3rd edn (Sinauer Associates, 2001).

29. Gabryś, H., Kramer, D. M., Crofts, A. R. \& Ort, D. R. Mutants of chloroplast coupling factor reduction in Arabidopsis. Plant Physiol. 104, 769-776 (1994).

30. Ünnep, R. et al. The ultrastructure and flexibility of thylakoid membranes in leaves and isolated chloroplasts as revealed by small-angle neutron scattering. Biochim. Biophys. Acta 1837, 1572-1580 (2014).

31. Garab, G., Kieleczawa, J., Sutherland, J. C., Bustamante, C. \& Hind, C. Organization of pigment-protein complexes into macrodomains in the thylakoid membranes of wild-type and chlorophyll b-less mutant of barley as revealed by circular dichroism. Photochem. Photobiol. 54, 273-281 (1991).

32. Garab, G. \& van Amerongen, H. Linear dichroism and circular dichroism in photosynthesis research. Photosynth. Res. 101, 135-146 (2009).

33. Johnson, M. P. \& Ruban, A. V. Rethinking the existence of a steady-state Deltapsi component of the proton motive force across plant thylakoid membranes. Photosynth. Res. 119, 233-242 (2014).

34. Wang, C., Yamamoto, H. \& Shikanai, T. Role of cyclic electron transport around photosystem $\mathrm{I}$ in regulating proton motive force. Biochim. Biophys. Acta 1847, 931-938 (2015).

35. Alonso, J. M. et al. Genome-wide insertional mutagenesis of Arabidopsis thaliana. Science 301, 653-657 (2003).

36. Rosso, M. G. et al. An Arabidopsis thaliana T-DNA mutagenized population (GABI-Kat) for flanking sequence tag-based reverse genetics. Plant Mol. Biol. 53, 247-259 (2003).

37. Robinson, S. J. et al. An archived activation tagged population of Arabidopsis thaliana to facilitate forward genetics approaches. BMC Plant Biol. 9, 101 (2009).

38. Karimi, M., De Meyer, B. \& Hilson, P. Modular cloning in plant cells. Trends Plant Sci. 10, 103-105 (2005).

39. Clough, S. J. \& Bent, A. F. Floral dip: a simplified method for Agrobacteriummediated transformation of Arabidopsis thaliana. Plant J. 16, 735-743 (1998).

40. Wu, F. H. et al. Tape-Arabidopsis Sandwich-a simpler Arabidopsis protoplast isolation method. Plant Methods 5, 16 (2009).

41. Irigoyen, S., Karlsson, P. M., Kuruvilla, J., Spetea, C. \& Versaw, W. K. The sinkspecific plastidic phosphate transporter PHT4;2 influences starch accumulation and leaf size in Arabidopsis. Plant Physiol. 157, 1765-1777 (2011).

42. Thuswaldner, S. et al. Identification, expression, and functional analyses of a thylakoid ATP/ADP carrier from Arabidopsis. J. Biol. Chem. 282, 8848-8859 (2007).

43. Fristedt, R. et al. Phosphorylation of photosystem II controls functional macroscopic folding of photosynthetic membranes in Arabidopsis. Plant Cell 21, 3950-3964 (2009).

44. Porra, R. J. The chequered history of the development and use of simultaneous equations for the accurate determination of chlorophylls a and b. Photosynth. Res. 73, 149-156 (2002)

45. Yin, L. et al. Role of thylakoid ATP/ADP carrier in photoinhibition and photoprotection of photosystem II in Arabidopsis. Plant Physiol. 153, 666-677 (2010).

46. Lichtenthaler, H. K. \& Welburn, A. R. Determinations of total carotenoids and chlorophylls a and b of leaf extracts in different solvents. Biochem. Soc. Trans. B 603, 591-592 (1983).
47. Genty, B., Briantais, J. M. \& Baker, N. R. The relationship between the quantum yield of photosynthetic electron transport and quenching of chlorophyll fluorescence. Biochim. Biophys. Acta 990, 87-92 (1989).

48. Schreiber, U. \& Klughammer, C. New accessory for the DUAL-PAM-100: The P515/535 module and examples of its application. PAM Appl. Notes 1, 1-10 (2008).

49. Cruz, J. A., Sacksteder, C. A., Kanazawa, A. \& Kramer, D. M. Contribution of electric field (Delta psi) to steady-state transthylakoid proton motive force (pmf) in vitro and in vivo. Control of pmf parsing into Delta psi and Delta $\mathrm{pH}$ by ionic strength. Biochemistry 40, 1226-1237 (2001).

50. Cruz, J. A. et al. Plasticity in light reactions of photosynthesis for energy production and photoprotection. J. Exp. Bot. 56, 395-406 (2005).

51. Checchetto, V. et al. Thylakoid potassium channel is required for efficient photosynthesis in cyanobacteria. Proc. Natl Acad. Sci. USA 109, 11043-11048 (2012).

52. Teuber, M., Rogner, M. \& Berry, S. Fluorescent probes for non-invasive bioenergetic studies of whole cyanobacterial cells. Biochim. Biophys. Acta 1506, 31-46 (2001)

53. Seigneurin-Berny, D., Salvi, D., Dorne, A. J., Joyard, J. \& Rolland, N. Percoll-purified and photosynthetically active chloroplasts from Arabidopsis thaliana leaves. Plant Physiol. Biochem. 46, 951-955 (2008).

54. Posselt, D. et al. Small-angle neutron scattering study of the ultrastructure of chloroplast thylakoid membranes-periodicity and structural flexibility of the stroma lamellae. Biochim. Biophys. Acta 1817, 1220-1228 (2012).

55. Nagy, G. et al. Kinetics of structural reorganizations in multilamellar photosynthetic membranes monitored by small-angle neutron scattering. Eur. Phys. J. E Soft Matter 36, 69 (2013).

56. Benson, D. A. et al. GenBank. Nucleic Acids Res. 41, D36-D42 (2013).

57. Bergsten, J. A review of long-branch attraction. Cladistics 21, 163-193 (2005)

58. Ronquist, F. et al. MrBayes 3.2: efficient Bayesian phylogenetic inference and model choice across a large model space. Syst. Biol. 61, 539-542 (2012).

59. Guindon, S. et al. New algorithms and methods to estimate maximumlikelihood phylogenies: assessing the performance of PhyML 3.0. Syst. Biol. 59, 307-321 (2010).

60. Hall, J. C., Sytsma, K. J. \& Iltis, H. H. Phylogeny of Capparaceae and Brassicaceae based on chloroplast sequence data. Am. J. Bot. 89, 1826-1842 (2002).

\section{Acknowledgements}

We thank Robert Last, Tomas Morosinotto, Giovanni Finazzi and Benoît Schoefs for feedback on this manuscript. We are grateful to Kathleen M. Imre (Michigan State University, East Lansing) for her help with Chloroplast 2010 project, Hans-Henning Kunz (Washington State University, Pullman) for his help with GFP localization constructs, the Centre for Cellular Imaging (Sahlgrenska Academy, Gothenburg University) for use of confocal and TEM microscopes and helpful assistance, Csilla Jónás (Eötvös Loránd University, Hungary) for embedding the TEM samples and Márton Markó (Wigner RC SZFI, Hungary) for discussion about analyses of SANS data. The authors are indebted to Joachim Kohlbrecher for help in configuring the SANS-I instrument and for interest in this project. We thank Eva-Mari Aro (Turku University) for the anti-D2 antibody and Torill Hundal (Stockholm University) for the anti-CP43 antibody. This work was supported by the Swedish Research Council and Enkvists Foundation (C.S.), Carl Tryggers Foundation (C.S., B.L.), PRIN 2010CSJX4F (I.S.), Human Frontiers Science Program RG0052 (I.S.), a Senior Postdoctoral Fellowship of the University of Padova (E.T.), Hungarian Research Fund OTKA, K112688 (G.G.) and the Sciex-Project 13.098 (R.Ü). A.K.N. was a recipient of a postdoctoral fellowship from Carl Tryggers Foundation. B.L. was partially supported by a grant from US National Science Foundation NSF MCB-1119778 to Robert Last (Michigan State University). Travel grants for presenting this work in international conferences were awarded by Helge Ax:Son Johnsons Foundation (A.H.) and University of Gothenburg Donationsnämnd (C.S.). The SANS part of this work was based on experiments undertaken at the Swiss spallation neutron source SINQ, Paul Scherrer Institute, Villigen, Switzerland.

\section{Author contributions}

A.H., C.S. and B.L. conceived the study; A.H., G.G., I.S., C.S. and B.L. designed the experiments. A.H. carried out chlorophyll $a$ fluorescence and ECS measurements. E.T. and I.S. undertook electrophysiology and acridine orange measurements. A.K.N. carried out localization experiments and quantitative PCR. B.E.P. conducted phylogenetic analyses. O.N.J., B.L. and K.S. carried out TEM analyses. R.Ü. and G.N. undertook SANS analyses. O.Z. carried out CD analyses. S.D. conducted RT-PCR. B.L. carried out immunoblotting. A.H., A.K.N., B.E.P., G.G., I.S. and C.S. wrote the manuscript. All authors helped to edit the manuscript.

\section{Additional information}

Supplementary Information accompanies this paper at http://www.nature.com/ naturecommunications

Competing financial interests: The authors declare no competing financial interests 
Reprints and permission information is available online at http://npg.nature.com/ reprintsandpermissions/

How to cite this article: Herdean, A. et al. A voltage-dependent chloride channel finetunes photosynthesis in plants. Nat. Commun. 7:11654 doi: 10.1038/ncomms11654 (2016). (c) (i) This work is licensed under a Creative Commons Attribution 4.0 International License. The images or other third party material in this article are included in the article's Creative Commons license, unless indicated otherwise in the credit line; if the material is not included under the Creative Commons license, users will need to obtain permission from the license holder to reproduce the material. To view a copy of this license, visit http://creativecommons.org/licenses/by/4.0/ 